\title{
A Case of Acute Pancreatitis Associated with Risperidone Treatment
}

\author{
Kentaro Kawabe, Shu-ichi Ueno \\ Department of Neuropsychiatry, Graduate School of Medicine, Ehime University, Ehime, Japan
}

\begin{abstract}
Acute pancreatitis with antipsychotic treatment is rare but sometimes causes a fatal adverse effect. Most cases of acute pancreatitis due to atypical antipsychotic agents are reported to occur within six months of starting antipsychotic administration. Acute pancreatitis caused by risperidone is rare. The patient had a high fever, stomachache and vomiting. The results of the abdominal computed tomograhpy scan were negative. The results of the abdominal ultrasonography were positive for gallstones in gallbladder and distention of the common bile duct. She had been fasting and received antibiotic intravenous injections. Amylase and lipase titers were high. After risperidone discontinuation, both the levels of the amylase and the lipase were gradually decreased. Three months later, the patient still maintains a good clinical balance. Although atypical antipsychotic-induced pancreatitis has been reported in conjunction with hyperglycemia, the pathophysiologic mechanism of these adverse events remains unclear. This case got pancreatitis 6 month after risperidone treatment. Using the antipsychotic agents, it is necessary to monitor pancreas function.
\end{abstract}

KEY WORDS: Schizophrenia; Risperidone; Aripiprazole; Pancreatitis,

\section{INTRODUCTION}

Acute pancreatitis due to antipsychotic treatment is rare but sometimes causes a fatal adverse effect. Some atypical antipsychotic agents, including clozapine, olanzapine, quetiapine, and risperidone, are associated with acute pancreatitis. ${ }^{1,2)}$ Among them, acute pancreatitis caused by risperidone is the rarest. ${ }^{3,4)}$ Although most cases of acute pancreatitis due to atypical antipsychotic agents occur within 6 months of starting antipsychotic administration, ${ }^{1)}$ we experienced a schizophrenic patient suffering from pancreatitis after more than 6 months of risperidone therapy.

\section{CASE}

A 69-year-old Japanese woman was diagnosed with schizophrenia at the age of 30 years and received outpatient care at another mental hospital. Her positive symptoms were not prominent, but her cognitive level was so impaired that she could not regulate her appetite and con-

\footnotetext{
Received: July 31, 2013 / Revised: September 3, 2013

Accepted: September 11, 2013

Address for correspondence: Kentaro Kawabe, MD

Department of Neuropsychiatry, Graduate School of Medicine,

Ehime University, Shitsukawa, Toon city, Ehime 791-0295, Japan

Tel: +81-89-960-5315, Fax: +81-89-960-5317

E-mail: k_kentaro_k@yahoo.co.jp
}

sumed about 2,000 kcal/day in addition to three ordinary meals. She had never smoked, did not drink alcohol, and did not take any illegal drugs. She underwent a hemigastrectomy, but the exact cause was unknown. She had been taking $2 \mathrm{mg} /$ day risperidone for more than 6 months. A neurological examination showed extrapyramidal stiffness. Blood tests were conducted repeatedly, but no abnormalities were detected. She developed a high fever, stomachache, and vomiting. We suspended the risperidone to improve her general condition. She was hospitalized for further investigation 2 days later. Blood tests (Table 1), abdominal ultrasonography, and a computed tomography (CT) scan were performed. Clinical features were accompanied by laboratory findings of hyperamylasemia (amylase, 1,191 U/L), hyperlipasemia (lipase, 1,514 U/L), and mild liver enzyme elevations. Creatine kinase (CK) was within the normal range. Blood pressure was stable and within the normal range. Results of the abdominal CT scan were negative for pancreatic and hepatic abnormalities. Results of the abdominal ultrasonography were positive for gallstones in the gallbladder and distention of the common bile duct. She had been fasting and received intravenous antibiotic injections. Subsequently, the amylase and lipase titers remained high ( $461 \mathrm{U} / \mathrm{L}$ and $804 \mathrm{U} / \mathrm{L}$, respectively), although alanine and aspartate aminotransferases decreased gradually to normal levels.

(c) This is an Open-Access article distributed under the terms of the Creative Commons Attribution Non-Commercial License (http://creativecommons.org/licenses/by-nc/3.0) which permits unrestricted non-commercial use, distribution, and reproduction in any medium, provided the original work is properly cited. 
Table 1. Course of the blood test

\begin{tabular}{lccrrrr}
\hline \multicolumn{1}{c}{ Time point } & Date of admission & Day 2 & Day 6 & Day 8 & Day 13 & Normal range \\
\hline Amylase (U/L) & 1,191 & 461 & 745 & 605 & 309 \\
Lipase (U/L) & 1,514 & 804 & 1,275 & 1,654 & 542 \\
AST (U/L) & 111 & 49 & 34 & 23 & 124 \\
ALT (U/L) & 238 & 166 & 81 & 51 & 27 \\
CPK (U/L) & 48 & 32 & 766 & 399 & $3-49$ \\
Risperidone (mg/day) & 2 & 0 & 2 & 0 & $45-226$ \\
\hline
\end{tabular}

AST, aspartate aminotransferase; ALT, alanine aminotransferase; CPK, creatine phosphokinase.

She did not have a painful or tender stomach. At this point, we felt that it was safe for her to start taking the risperidone again.

Two days after starting the risperidone, serum lipase and amylase increased again to $1,275 \mathrm{U} / \mathrm{L}$ and $745 \mathrm{U} / \mathrm{L}$, respectively, and CK also increased (766 U/L). No neurological or physical signs were detected. We decided to suspend the risperidone and introduced $10 \mathrm{mg}$ intravenous haloperidol injections once per day.

Two days after discontinuing the risperidone, the serum amylase decreased $(605 \mathrm{U} / \mathrm{L})$, but the serum lipase level remained elevated $(1,654 \mathrm{U} / \mathrm{L})$. One week after discontinuing the risperidone, the levels of amylase and lipase decreased gradually $(309 \mathrm{U} / \mathrm{L}$ and $542 \mathrm{U} / \mathrm{L}$, respectively), and CK dropped to the normal range. As her general clinical condition and biochemical markers were stable, we changed the haloperidol injection to an oral solution of $6 \mathrm{mg} /$ day aripiprazole because her mental condition worsened after stopping the risperidone treatment. Her mental status improved with the aripiprazole treatment, and she was discharged without positive laboratory findings. The patient's monthly blood tests continue to be normal, including amylase, lipase, and blood cell counts.

\section{DISCUSSION}

Although atypical antipsychotic-induced pancreatitis has been reported in conjunction with hyperglycemia, ${ }^{5)}$ the pathophysiological mechanism of these adverse events remains unclear. Most antipsychotic-induced pancreatitis occurs within 6 months after administration ${ }^{1)}$; however, our case developed pancreatitis more than 6 months after the start of risperidone treatment. Serotonin is associated with the development and aggravation of acute pancreatitis. Risperidone is a 5-HT2A antagonist and ameliorates diet-induced necrotic pancreatitis in mice, ${ }^{6)}$ and reduced serum pancreatic amylase levels is observed after endoscopic retrograde cholangiopancreatography. ${ }^{7)}$ However, there is no evidence of an association between risperidone treatment and acute pancreatitis. A thorough evaluation for pancreatitis, such as alcohol, tumor, and autoimmune causes, was completed in this case. Gallstones were present, which were due to an adverse effect of risperidone because the two separate risperidone administrations elevated serum amylase and lipase independently. Aripiprazole is currently used in such cases, as aripiprazole is thought to have fewer effects on metabolism, including saccharometabolism, than other atypical antipsychotic agents. Lifestyle was also a risk factor in this case. Thus, it is necessary to monitor pancreatic function in addition to hyperglycemia in such cases.

\section{REFERENCES}

1. Koller EA, Cross JT, Doraiswamy PM, Malozowski SN. Pancreatitis associated with atypical antipsychotics: from the Food and Drug Administration's MedWatch surveillance system and published reports. Pharmacotherapy 2003;23: 1123-1130.

2. Gropper D, Jackson CW. Pancreatitis associated with quetiapine use. J Clin Psychopharmacol 2004;24:343-345.

3. Berent I, Carabeth J, Cordero MM, Cordero R, Sugerman $\mathrm{B}$, Robinson D. Pancreatitis associated with risperidone treatment? Am J Psychiatry 1997;154:130-131.

4. Cordeiro Q Jr, Elkis H. Pancreatitis and cholestatic hepatitis induced by risperidone. J Clin Psychopharmacol 2001;21: 529-530.

5. Reddymasu S, Bahta E, Levine S, Manas K, Slay LE. Elevated lipase and diabetic ketoacidosis associated with aripiprazole. JOP 2006;7:303-305.

6. Yamaguchi I, Hamada K, Yoshida M, Isayama H, Kanazashi S, Takeuchi K. Risperidone attenuates local and systemic inflammatory responses to ameliorate diet-induced severe necrotic pancreatitis in mice: it may provide a new therapy for acute pancreatitis. $J$ Pharmacol Exp Ther 2009;328:256262.

7. Uchino R, Isayama H, Tsujino T, Sasahira N, Ito Y, Matsubara S, et al. Results of the Tokyo Trial of Prevention of Post-ERCP Pancreatitis with Risperidone-2: a multicenter, randomized, placebo-controlled, double-blind clinical trial. Gastrointestinal Endoscopy 2013. [Epub ahead of print] 